\title{
Volume optimization of gear trains with spur gears using genetic algorithm
}

\author{
Nenad Marjanović ${ }^{1}$, Nenad Kostić ${ }^{1,{ }^{*}}$, Nenad Petrović$^{1}$, Mirko Blagojević ${ }^{1}$, and Miloš \\ Matejić ${ }^{1}$ \\ ${ }^{1}$ University of Kragujevac, Faculty of Engineering, 6 Sestre Janjić, 34000 Kragujevac, Serbia
}

\begin{abstract}
Gear train volume optimization presents a complex problem tied to practical application in gear train manufacturing. This paper is oriented on the analysis of the problem of gear train volume minimization from a shaft axes positioning aspect. An original mathematical model has been developed where the objective function gives a minimum volume with changed shaft (spur gear) axes positions, while at the same time complying with all physical constraints. An original optimization software has also been developed using RCGA (Real Coded Genetic Algorithm) optimization methods. The general mathematical model was applied to three real conceptions of gear train as well as a comparative analysis of initial and optimal values. The results show a decrease of volume being directly linked to a decrease of not only space but material used to make the housing, costs, documentation formulation rate, etc.
\end{abstract}

\section{Introduction}

Gear train presents a complex technical system due to the complexity of its mathematical formulation and large number of variable values. From a research and practical aspect there is a need for constant improvement theoretical and practical segments in order to justify gear train application. Achieving better operational gear train characteristics, and a lower mass, volume and cost are basic objectives when designing a gearbox. Optimization is the most effective way of achieving desired gear train characteristics, it allows for advances in this field as a current and alternative method of improvement. This paper only considers parallel shaft axes gear train.

The optimization method which has proven itself as significant for solving practical problems is the heuristic method of genetic algorithm, which is why it is interesting for this field of research. In this specific case the RCGA (Real Coded Genetic Algorithm) was developed, dedicated to solving general engineering problems.

Aside from knowing the construction of gear train, the process, and method of optimization, the development of a mathematical model is necessary which can be representative of the problem and improve gear train performance. This paper contains relevant examples which can present results of performance improvements with gear trains.

\footnotetext{
*Corresponding author: nkostic@kg.ac.rs
} 
Chonget al. [1] presented a general methodology for optimizing gear ratios, sizes and housing volume for multi-stage gear trains in preliminary design phases. Marjanovic et al. [2] developed a practical approach to optimizing gear trains with spur gears based on selection matrix, selection of optimal materials, optimal gear ratios and shaft axes positions. Golabiet. Al [3] presented gear train volume/weight minimization optimizing single and multistage gear trains' gear ratios. Mendi et al. [4] aimed to optimize gear train component dimensions to achieve minimal volume comparing GA results to analytic method parameter volume. Savsani et al. [5] described gear train weight optimization comparing various optimization methods to genetic algorithm (GA) result values. Gologlu and Zeyveli [6] performed preliminary design automation through optimization of gear parameters and properties using a GA based approach. Tand and Tseng [7] presented a new mutation operator to adaptive direct mutation that focuses on simplicity, robustness, and efficiency within the context of RCGAs. Shopova et al. [8] presented an adjustable genetic algorithm deigned to deal with engineering optimization problems with both real and integer problems. Mushavati and Hamouda [9] implemented cyclic crossover operation to modify GA for multiple part manufacturing line optimization. For the manufacturing process optimization it is necessary to employ theoretical models as presented in [11]. Such a process is practical [12], economical [13], and relatively easy for use [14].

There is a real need to explain and mathematically formulate the gear train volume optimization problem, to find influential factors, and to find approaches and methods through which optimal gear train characteristics are possible.

Motivation is based on gear train performance; achieving a minimum volume, consequently a smaller mass, smaller embedded measurements, less material used, decreased expenses and other benefits.

The results obtained by the here presented method are compared to common concepts through specific examples.

\section{Problem definition}

Gear trains with spur gears are most frequently made so their shaft axes are in the same plane, which is most frequently the horizontal plane.

Gears placed in this way take up a large volume which can be reduced through optimizing the transmission. An optimal solution defines a new position of gear train shaft axes which results in a decreased overall volume of the gear train [15-16].

Gear train volume can be defined as a result of multiplying length, height, and width based on which a mathematical model can be made. Since width considered as a constant due to calculated values of spur gears and other components' constructive dimensions, the optimization problem becomes planar, based on optimizing the area defined by multiplying length $(L)$, and width $(H)$ of the transmission.

Fig. 1 shows the positions of the gears relative to the $\mathrm{x}$ and $\mathrm{y}$ axes. The position of the input shaft is constrained in the origin, while the positions of other shafts is defined by the angle, $\theta_{i}$ which is also shown in Fig.1. Gearbox volume is directly dependent on the change of these angles. A constant, $c_{l}$, is left on all sides as a technical clearance and is included in both the initial and optimal models, which is added to form the overall length and height. 


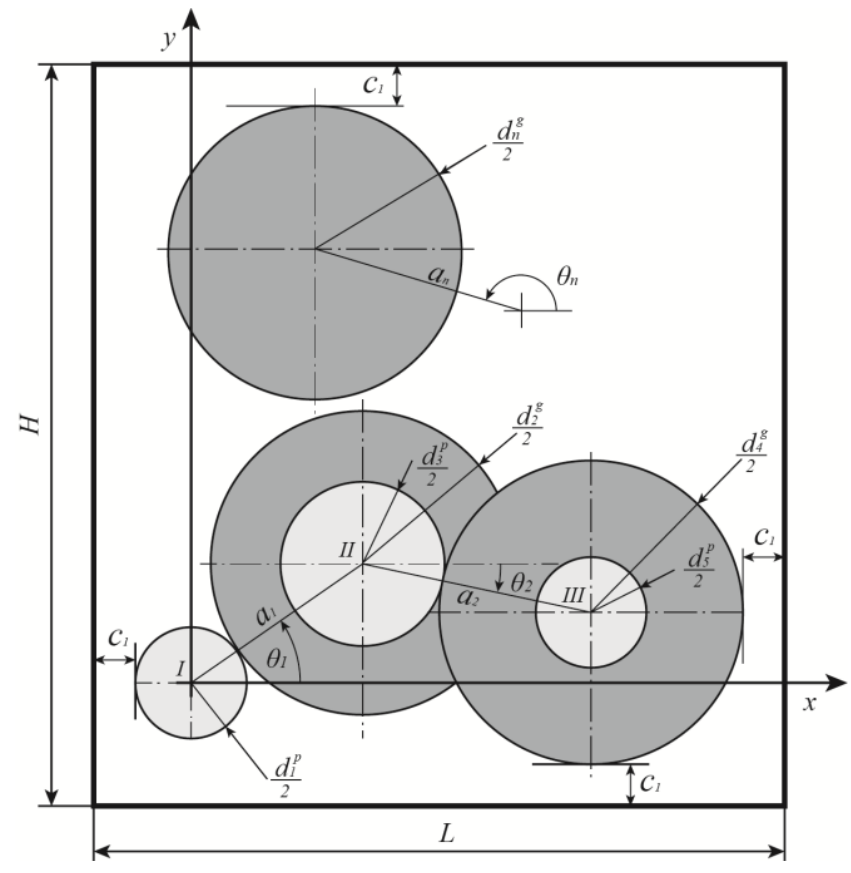

Fig. 1. Formulation of optimal gear train shaft axes.

Before optimization, in the initial design phase the following condition must be met for paired gears $u$ and $v$ in cases of gear trains with three or more stages:

$$
a_{u v}>\frac{d_{u-1}^{p}}{2}+\frac{d_{v-1}^{g}}{2}
$$

This ensures there is no clash between non paired elements placed in the same plane. If more than one stage can be placed in the same plane, this will initially give a decrease of volume even before optimization (Fig. 2a).

\section{Mathematical model}

Once all initial design conditions are met, and gear dimensions calculated, the typically positioned gear train can be optimized. Gear train volume changes when shaft position angles are changed. Minimum volume is achieved through achieving a minimal area of the profile plane. A difference of the rightmost coordinate of the gear train from the origin placed in the first pinion, and the leftmost coordinate from the origin gives the length of the gear train for the observed angles. In the same way the topmost, and bottom most coordinates subtracted give the height of the gear train. The function to determine the area which the gear train occupies is defined as follows:

$$
\begin{aligned}
& f=\left[\max \left(\frac{d_{a i}^{(p)}}{2}, \sum_{i=1}^{n}\left(a_{i} \cdot \cos \theta_{i}\right)+\frac{d_{a i}^{(g)}}{2}\right)+c_{1}-\min \left(-\frac{d_{a i}^{(p)}}{2}, \sum_{i=1}^{n}\left(a_{i} \cdot \cos \theta_{i}\right)-\frac{d_{a i}^{(g)}}{2}\right)+c_{1}\right] . \\
& \cdot\left[\max \left(\frac{d_{a i}^{(p)}}{2}, \sum_{i=1}^{n}\left(a_{i} \cdot \sin \theta_{i}\right)+\frac{d_{a i}^{(g)}}{2}\right)+c_{1}-\min \left(-\frac{d_{a i}^{(p)}}{2}, \sum_{i=1}^{n}\left(a_{i} \cdot \sin \theta_{i}\right)-\frac{d_{a i}^{(g)}}{2}\right)+c_{1}\right] .
\end{aligned}
$$

This research presents a gear train optimization approach based on changing positions of gear shaft axes in relation to the horizontal plane. The angle between shaft angle positions can vary in the range of: $-180^{\circ} \leq \theta_{i} \leq 180^{\circ}$. The previous equation represents a 
general mathematical model. The equation includes the addition of $15 \mathrm{~mm}$ on all sides of the gear train area as technical clearance for a housing. This clearance is also included as a clearance on all sides in the horizontal plane, as well as being a minimal distance between two gears on a single shaft.

In order to properly constrain the complex mathematical model, so that the result of the optimization gives a realistic model, many design factors need to be considered. Multilevel gear trains with spur gears can be set up in different ways in the initial design phase depending on spur gear dimensions. In case two sets of gears can be placed in the same plane and maintain a minimal clearance between non-paired gears, the volume can be decreased initially by reducing the overall width of the gear train.

Optimization constraints, similarly to the initial condition (Fig. 2a), in this case are maintaining a minimal clearance between non-paired gears of these stages when the shaft axes angles change (Fig.2b). For all stages in separate planes there is an obligatory constraint for avoiding clash of shaft and gear, and limiting their distance to a minimal possible value of $15 \mathrm{~mm}$, where this clash is possible (Fig.2c).

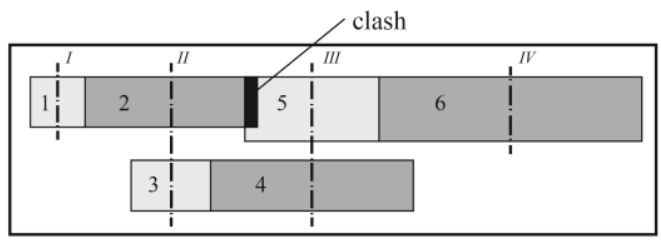

a)

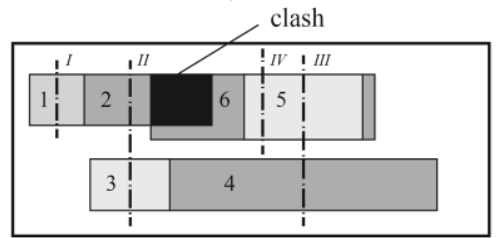

b)

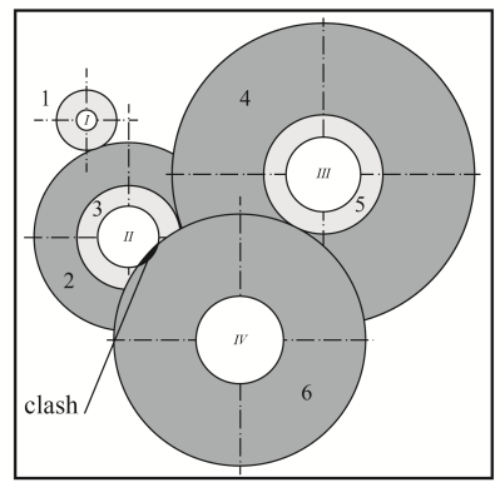

c)

Fig. 2. Possible clashes between a) non paired gears in initial design phase, b) non paired gears in optimized model, c) shaft and gear in optimized model.

The objective function for all concepts has an addition of a minimum distance between gears and housing on all sides. The minimal volume which is achieved through optimization includes clearances not only in the observed plane, but also on shaft lengths. These technical clearances have constant values.

Constraints which ensure against clash of any non-paired elements $m$ and $n$ are given as a minimal center distance between those elements which must be greater than or equal to the sum of their respective radiuses and a constant technical clearance. The general equation for this constraint is:

$$
\begin{aligned}
& a_{m n}=\sqrt{x_{m n}^{2}+y_{m n}^{2}} \geq \frac{d_{m}}{2}+\frac{d_{n}}{2}+c_{2} ; \quad c_{2}=15 m m \\
& x_{m n}=\sum_{j=m}^{n} a_{j} \cdot \cos \theta_{j} ; \quad y_{m n}=\sum_{j=m}^{n} a_{j} \cdot \sin \theta_{j} .
\end{aligned}
$$




\section{Optimization method and software}

Gear train volume optimization is a complex problem and as such is not possible without the use of a computer. In order to optimize, it is necessary to use an optimization method flexible for complex problems with numerous constraints. In this case, genetic algorithm method was chosen due to its favorable characteristics, and an original software has been developed for optimization based on this method. A real coded genetic algorithm (RCGA) was used, the algorithm is presented in Fig.3. The software used was developed in $\mathrm{C}++$.

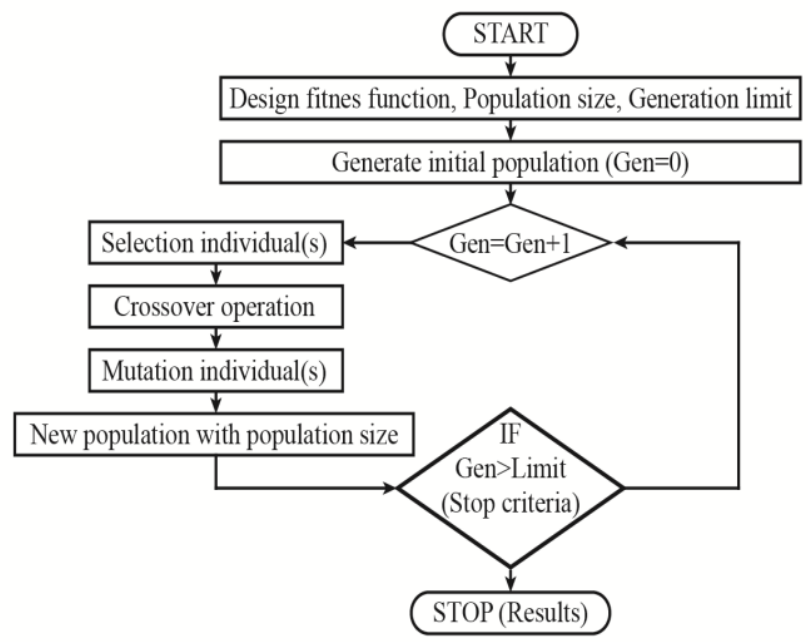

Fig. 3. Genetic algorithm for real coding (RCGA).

Genetic algorithm (GA) is a heuristic method for optimizing whose operation is based on mimicking natural/evolutionary processes [8]. The algorithm contains three basic operators: selection, crossover, and mutation. Selection is the process of transferring genetic information through generations. Crossover represents the process/operations between two parents, where an exchange of genetic information and new generations are made. The mutation operator creates a random change in the genetic structure of some individuals for overcoming early convergence. Algorithm operation is based on survival of the fittest individuals through evolution which exchange genetic material. Selection ranks individuals in the population using values from the fitness function, which defines the ability/quality of the individual.

Genetic algorithm, due to its convergence characteristics has a widespread application. Researchers are inspired to use this algorithm for scientific purposes, industrial application, business applications and to further increase its use.

Before use the software was tested according to available literature and its operation meets all necessary criteria. The original software is intended for all problems formulated by a mathematical model.

\section{Test examples}

For the purpose of verifying the mathematical model, in this paper three examples of various multistage gear trains were put through the optimization process. As the model can be applied to any gear train with two or more stages the examples used are of a two stage (Example 1) [2], three stage (Example 2) [2], and four stage (Example 3) [1] gear trains. Selected gear trains are representative of real practical examples used in the industry and 
are comparable to results from literature. The input values for the mathematical model for these examples are shown in Table 1.

Table 1 also gives initial gross dimensions of each example with included technical clearances. As the mathematical model aims to find the minimal volume by changing the area normal to shaft axes optimized examples are hypothesized to change in some measure correlating with the change of shaft position angles. Volumes of test examples in the initial concept arrangement are $9925631.1 \mathrm{~mm}^{3}$ for Example 1, $72341883 \mathrm{~mm}^{3}$ for Example2, and $39697456.5 \mathrm{~mm}^{3}$ for Example 3. The concept of Example 2 does not have the $1^{\text {st }}$ and $3^{\text {rd }}$ gear pairs in the same axes due to the initial design conditions not being met. Example 3 has the $4^{\text {th }}$ gear pair face aligned with the same side face of the $1^{\text {st }}$ gear pair which also places it behind the $2^{\text {nd }}$ gear pair due to the width of the $4^{\text {th }}$ gear pair. The initial concept of Example 3 therefore has an initially decreased volume than if the gear pairs were all in their own respective planes.

Table 1. Input values for creating a mathematical model for all examples.

\begin{tabular}{|c|c|c|c|c|c|c|c|c|c|c|}
\hline & \multicolumn{3}{|c|}{ Example 1(2 stage) } & \multicolumn{3}{|c|}{ Example 2 (3 stage) } & \multicolumn{4}{|c|}{ Example 3 (4 stage) } \\
\hline Stage & 1 & 2 & & 1 & 2 & 3 & 1 & 2 & 3 & 4 \\
\hline $\begin{array}{l}\text { Module } \\
(\mathrm{mm})\end{array}$ & 2 & 3 & & 2 & 3 & 4.5 & 1.5 & 2 & 3 & 4 \\
\hline $\begin{array}{l}\text { Number } \\
\text { of teeth in } \\
\text { pinion }\end{array}$ & 20 & 23 & & 24 & 24 & 23 & 14 & 18 & 20 & 25 \\
\hline $\begin{array}{l}\text { Number } \\
\text { of teeth in } \\
\text { gear }\end{array}$ & 83 & 67 & & 110 & 84 & 56 & 77 & 79 & 74 & 82 \\
\hline $\begin{array}{c}\text { Pitch } \\
\text { diameter } \\
\text { of pinion } \\
(\mathrm{mm})\end{array}$ & 40 & 69 & & 48 & 72 & 103.5 & 21 & 36 & 60 & 100 \\
\hline $\begin{array}{l}\text { Pitch } \\
\text { diameter } \\
\text { of gear } \\
(\mathrm{mm})\end{array}$ & 166 & 201 & & 220 & 252 & 252 & 115.5 & 158 & 222 & 328 \\
\hline $\begin{array}{c}\text { Face } \\
\text { width } \\
(\mathrm{mm})\end{array}$ & 103 & 135 & & 134 & 162 & 177.75 & 68.25 & 97 & 141 & 214 \\
\hline $\begin{array}{c}\text { Net } \\
\text { length, } \\
\text { height, } \\
\text { width }\end{array}$ & 388.5 & 231 & 110.6 & 653.75 & 282 & 392.4 & 724.75 & 358 & 153 & \\
\hline
\end{tabular}

\section{Results}

The mathematical model is formed based on geometric characteristics of multistage gear trains. Optimization of initial concepts was conducted in the aforementioned software using RCGA. Input values were inserted into the mathematical model from Table 1.

As the width of the gear train is unchanged, the mathematical model gives a minimal area. Convergence of the area and shaft axes angles are shown in Figures 4, 5, and 6 for a smaller number of iteration steps to illustrate rate of convergence. Optima were achieved in 1500 iteration steps for Example 1, 3000 for Example 2, and 6000 for Example 3. 


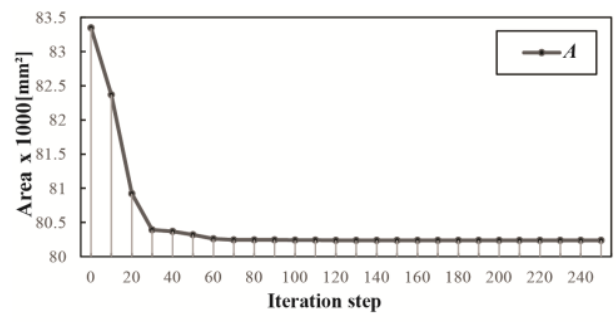

a)

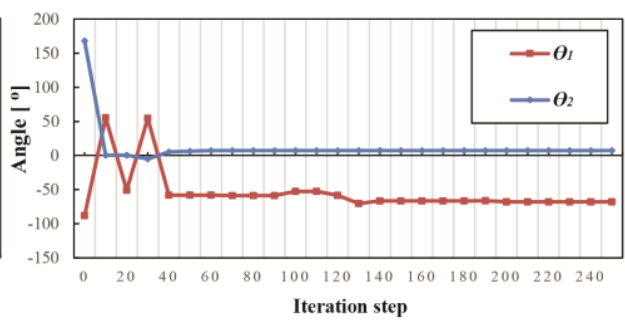

b)

Fig. 4. Example1 convergence of a) minimal area, b) angle values, at iteration step.

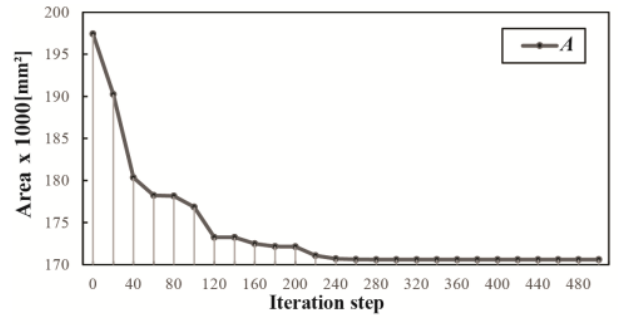

a)

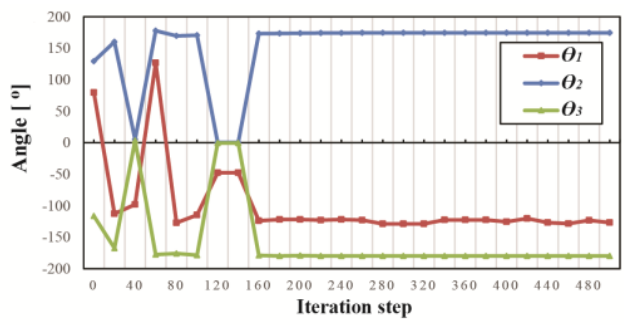

b)

Fig. 5. Example 2 convergence of a) minimal area, b) angle values, at iteration step.

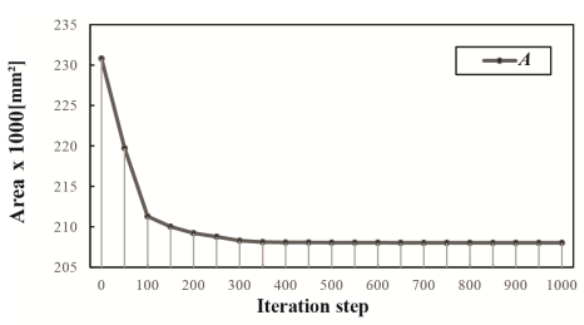

a)

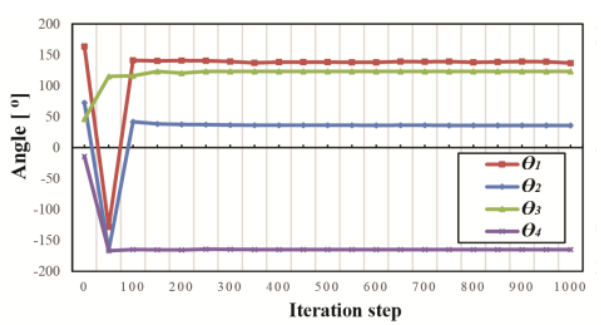

b)

Fig. 6. Example 3 convergence of a) minimal area, b) angle values, at iteration step.

The optimization results shown in Table 2 show an overall decrease in volume for all examples. Examples 1 and 2 show an unchanged height and decreased length, while Example 3 has an increased height and significantly decreased height.

Table 2. Optimal values of shaft position angles, overall dimensions, and volume for examples.

\begin{tabular}{|c|c|c|c|}
\hline & Example 1 & Example 2 & Example 3 \\
\hline$\theta_{1}\left[{ }^{\circ}\right]$ & -55.0359 & -61.3595 & -154.2624 \\
\hline$\theta_{2}\left[{ }^{\circ}\right]$ & 7.4482 & 5.6681 & -144.9345 \\
\hline$\theta_{3}\left[{ }^{\circ}\right]$ & - & 0 & -57.7319 \\
\hline$\theta_{4}\left[{ }^{\circ}\right]$ & - & - & 14.3393 \\
\hline $\mathrm{L}[\mathrm{mm}]$ & 347.361 & 604.958 & 555.61 \\
\hline $\mathrm{H}[\mathrm{mm}]$ & 231 & 282 & 373.702 \\
\hline $\mathrm{B}\left[\mathrm{mm}^{3}\right]$ & 110.6 & 392.4 & 153 \\
\hline $\mathrm{V}\left[\mathrm{mm}^{3}\right]$ & 8874585.592 & 66942709.8 & 31767763.44 \\
\hline
\end{tabular}

Initial angles have changed significantly from original concepts with all shaft axes in the same plane resulting in optimal concepts with a minimal volume (Fig. 7). 

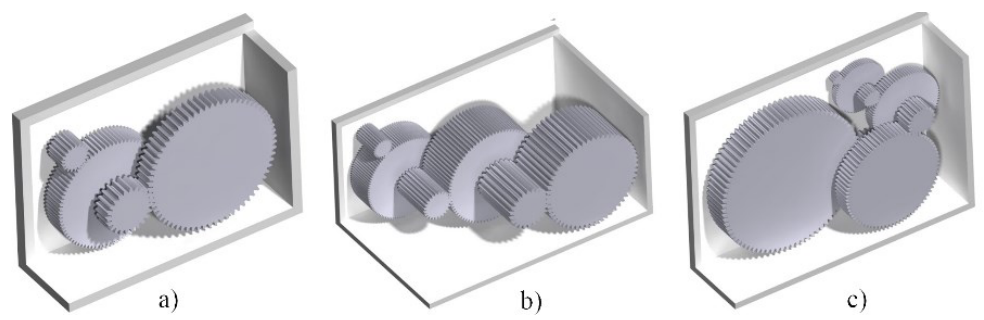

Fig. 7. Optimal concept positions for a) Example 1, b) Example 2, and c) Example 3.

Examples in figure 7 are given as a section view of a simplified housing in order to better show the optimal interior volumes and concepts of the gear trains. Example 3 in figure 6 is shown with the housing rotated by $90^{\circ}$ for better visualization.

Unlike in [1] constraints for Example 3, as well as for all other examples, do not allow for an overlap of shafts and gears. All shafts have an intended clearance to allow for shafts to be designed in any desired length without forcing the design of a more complex housing to compensate for shaft embedding.

Example 1 gives an optimal area (volume) for the range of $-52.4912^{\circ} \leq \theta_{1} \leq$ $-72.0083^{\circ}$. All concepts also give optimal volumes for completely mirrored or completely normal values of all angle optima.

\section{Conclusions}

Optimization of gear trains with spur gears is a complex process. One such practical approach is presented in this paper. Presented in the paper is a universal mathematical model which can be applied to gear trains with two or more stages. Three examples are shown optimized using the presented mathematical model calculated in an original software based on a GA method. Real coded genetic algorithm (RCGA) is used for optimization. A large number of iterations is necessary to find optima due to the complexity of the mathematical model.

The examples initial volumes are calculated for the common case where all shaft axes are in the same plane. Comparison of initial volumes and optimization results for concepts of Example 1 show a decrease in volume of $10.589 \%$, for Example 2 a decrease of $7.463 \%$, and for Example 3 a decrease of $19.975 \%$.

Gear trains with 2, 3 and 4 stages are used as test examples to show the influence of the number of stages on the percentage of volume decrease. Example 2 is used to show that the number of stages does not directly influence volume minimization. In other words the twostage gear train (Example 1) has a greater relative decrease in volume from its original conception than the three-stage gear train (Example 2) due to a small difference in pinion sizes relative to their corresponding gears. If pinion to gear ratios are greater the area, and in turn volume is expected to have a greater difference in volume for optimal shaft positions.

Initial conditions and optimization constraints are incorporated to ensure the optimal concepts are practically applicable. The achieved minimization of volume in turn results in lower costs and weights of the housing for such gear trains.

\section{References}

1. T.H. Chong, I. Bae, G.J. Park, MMT, 37, 295 (2002)

2. N. Marjanovic, B. Isailovic, V. Marjanovic, Z. Milojevic, M. Blagojevic, M. Bojic, MMT, 53, 1 (2012) 
3. S.I. Golabi, J.J. Fesharaki, M. Yazdipoor, Mechanism and Machine Theory, 73, 197 (2014)

4. F. Mendi, T. Başkal, K. Boran, F.E. Boran, ESA, 37, 8058 (2010)

5. V. Savsani, R.V. Rao, D.P. Vakharia, MMT, 45, 531 (2010)

6. C. Gologlu, M. Zeyveli, Computers \& Industrial Engineering, 57, 1043 (2009)

7. P.H. Tang, M.H. Tseng, Applied Soft Computing, 13, 600 (2013)

8. E.G. Shopova, N.G. Vaklieva-Bancheva, Computers \& Chemical Engineering, 30, 1293 (2006)

9. F. Musharavati, A.S.M. Hamouda, ESA, 38, 10770 (2011)

10. S. Wojciechowski, R. Maruda, P. Nieslony et al., International Journal of Mechanical Sciences, 119, 360 (2016)

11. R. Maruda, E. Feldshtein, et al., AJSE, 41, 661 (2016)

12. J.B. Krolczyk, G.M. Krolczyk, S. Legutko, et al, Technical Gazette, 22, 1447 (2015)

13. G.M. Krolczyk, S. Legutko, MMS, 21, 759 (2014)

14. G.R.S. X. Li, G. Cockerham, MMT, 31, 717 (1996)

15. S.P.N. Mushtaq M.A. Kader, G.K. Grover, MMT, 33, 839 (1998) 